\title{
EXTREME POINTS OF CERTAIN TRANSPORTATION POLYTOPES WITH FIXED TOTAL SUMS*
}

\author{
ZHI CHEN ${ }^{\dagger}$, ZELIN ZHU ${ }^{\ddagger}$,JIAWEI LI ${ }^{\ddagger}$, LIZHEN YANG ${ }^{\ddagger}$, AND LEI CAO ${ }^{\S}$
}

\begin{abstract}
Transportation matrices are $m \times n$ nonnegative matrices with given row sum vector $R$ and column sum vector $S$. All such matrices form the convex polytope $\mathcal{U}(R, S)$ which is called a transportation polytope and its extreme points have been classified. In this article, we consider a new class of convex polytopes $\Delta(\bar{R}, \bar{S}, \sigma)$ consisting of certain transportation polytopes satisfying that the sum of all elements is $\sigma$, and the row and column sum vectors are dominated componentwise by the given positive vectors $\bar{R}$ and $\bar{S}$, respectively. We characterize the extreme points of $\Delta(\bar{R}, \bar{S}, \sigma)$. Moreover, we give the minimal term rank and maximal permanent of $\Delta(\bar{R}, \bar{S}, \sigma)$.
\end{abstract}

Key words. Transportation polytopes, Extreme points, Term ranks, Permanents.

AMS subject classifications. 15B51, 52B05.

1. Introduction. Let $R=\left(r_{1}, \ldots, r_{m}\right)$ and $S=\left(s_{1}, \ldots, s_{n}\right)$ be nonnegative vectors satisfying

$$
\sigma=\sum_{i=1}^{m} r_{i}=\sum_{j=1}^{n} s_{j}
$$

The transportation polytope $\mathcal{U}(R, S)$ is the set of all $m \times n$ nonnegative matrices with row sum vector $R$ and column sum vector $S$. The matrices in $\mathcal{U}(R, S)$ are called transportation matrices. Transportation polytopes model the transportation of goods from $m$ supply locations to $n$ demand locations. The $i$ th supply location supplies a quantity of $r_{i}$, while the $j$ th demand location demands a quantity of $s_{j}$. A matrix $A=\left[a_{i, j}\right]$ in $\mathcal{U}(R, S)$ models the scenario where $a_{i, j}$ is the amount of material transported from the $i$ th supply location to the $j$ th demand location. The $i$ th row sum of $A$ is denoted by $r_{i}(A)$, and the $j$ th column sum of $A$ is denoted by $s_{j}(A)$. Denote by $R(A)$ the row sum vector of a matrix $A$ and by $S(A)$ the column sum vector of $A$, i.e.

$$
R(A)=\left(r_{1}(A), \ldots, r_{m}(A)\right), S(A)=\left(s_{1}(A), \ldots, s_{n}(A)\right) .
$$

The polytope $\mathcal{U}(R, S)$ is nonempty if and only if (1.1) holds. Due to the applications in many optimization problems, transportation polytopes have been intensively studied by many mathematicians, e.g. [3, 17, 20, 16, 13, 15]. Given an $m \times n$ matrix $A$, let $\mathcal{P}(A)$ denote the $(0,1)$-matrix with 1 's in the position of the nonzero entries of $A$ and 0 's elsewhere. $\mathcal{P}(A)$ is called the pattern of $A$. The bipartite graph $B G(A)$ of $A$ is a graph with vertex set $\left\{\mathbf{r}_{1}, \ldots, \mathbf{r}_{m}\right\} \cup\left\{\mathbf{s}_{1}, \ldots, \mathbf{s}_{n}\right\}$, where there is an edge between $\mathbf{r}_{i}$ and $\mathbf{s}_{j}$ if and only if

\footnotetext{
* Received by the editors on January 9, 2020. Accepted for publication on June 26, 2020. Handling Editor: Bryan Shader. Corresponding Author: Zhi Chen.

${ }^{\dagger}$ Department of Mathematics, Nanjing Agricultural University, Jiangsu 210095, China (chenzhi@njau.edu.cn). Supported by the National Natural Science Foundation of China (No. 11601233); The Natural Science Foundation of Jiangsu Province (BK20160708); The Fundamental Research Funds for the Central Universities (No. KJQN201718).

${ }^{\ddagger}$ Department of Mathematics, Nanjing Agricultural University, Jiangsu 210095, China. Lizhen Yang, Zelin Zhu and Jiawei Li are supported by the Student Research Training Program (No. 1923A09).

$\S$ Department of Mathematics, Halmos College, Nova Southeastern University, Fort Lauderdale, FL, 33314, USA.
} 
$a_{i, j} \neq 0$. A line of a matrix $A$ means either a row or a column of $A$. Denote the set of extremal matrices of $\mathcal{U}(R, S)$ by $\mathfrak{E}(R, S)$, which was first investigated by Jurkat and Ryser in [17].

We summarize the results in the following Proposition.

Proposition 1.1 ([12, 17]). If $A \in \mathcal{U}(R, S)$, then the following conditions are equivalent:

(i) $A \in \mathfrak{E}(R, S)$.

(ii) Every submatrix of $A$ contains a line with at most one positive entry.

(iii) Every submatrix $A^{\prime}$ of $A$ of size $m^{\prime} \times n^{\prime}$ has at most $m^{\prime}+n^{\prime}-1$ positive entries.

(iv) There is no matrix $B \in \mathcal{U}(R, S)$ such that $B \neq A$ and $\mathcal{P}(B)=\mathcal{P}(A)$.

(v) $B G(A)$ is a forest with no isolated vertex.

Given nonnegative vectors $R=\left(r_{1}, \ldots, r_{m}\right)$ and $S=\left(s_{1}, \ldots, s_{n}\right)$ satisfying $(1.1)$, denote by $\mathcal{U}_{\leq}(R, S)$ the convex set of all $m \times n$ nonnegative matrices with row sum vectors dominated componentwise by $R$ and column sum vectors dominated componentwise by $S$. We say that the $i$ th row sum (resp. $j$ th column sum) of a matrix $B$ in $\mathcal{U}_{\leq}(R, S)$ is unattained if $r_{i}(B)<r_{i}$ (resp. $s_{j}(B)<s_{j}$ ). Equivalently, we also say the $i$ th row (resp. $j$ th column) vertex in $B G(B)$ is unattained. Denote the set of all extreme points in $\mathcal{U}_{\leq}(R, S)$ by $\mathfrak{E}_{\leq}(R, S)$. In [4], Brualdi gave a combinatorial classification of the extreme points of $\mathcal{U}_{\leq}(R, S)$ as the following theorem.

Proposition 1.2 ([4]). If $B \in \mathcal{U}_{\leq}(R, S)$, then the following conditions are equivalent:

(i) $B \in \mathfrak{E}_{\leq}(R, S)$.

(ii) $B G(B)$ is a forest where at most one vertex of each tree corresponds to a row or a column of $B$ whose sum in $B$ is unattained.

(iii) There exists some extreme point $A$ of $\mathcal{U}(R, S)$, such that $B G(B)$ can be obtained by deleting a set (possibly empty) of edges of a subtree from each connected components in $B G(A)$. Thus $B$ can be obtained by replacing by zero the positive entries of $A$ corresponding to the deleted edges.

By specializing $m=n$ and $R=S=(1, \ldots, 1) \in \mathbb{R}^{n}$ in $\mathcal{U}(R, S)$, we obtain the convex polytope $\Omega_{n}$ of all $n \times n$ doubly stochastic matrices. The extreme points of $\Omega_{n}$ were characterized by Birkhoff [1] to be all $n \times n$ permutation matrices. Mirsky [23] investigated the convex polytope $\omega_{n}$ of all $n \times n$ doubly substochastic matrices which are nonnegative matrices whose row and column sums are at most 1 . He proved that the extreme points of $\omega_{n}$ are all $n \times n$ subpermutation matrices. In [18] and [19], Katz gave the extreme points of the polytopes of symmetric doubly stochastic matrices and symmetric doubly substochastic matrices, respectively. In 1977, Cruse [7] characterized the extreme points of the polytope of centrosymmetric doubly stochastic even matrices, while the odd case was solved by Brualdi and Cao [6] in 2018. Later on, Chen, Cao and Wang [11] characterized the extreme points of the polytope of centrosymmetric doubly substochastic matrices. Moreover, Cao and Chen [9] studied the convex set $\omega_{n}^{s}$ of all $n \times n$ doubly substochastic matrices with the sum of all elements equal to $s$.

For a positive integer $n$ and $0 \leq s \leq n$, denote by $\lfloor s\rfloor$ the greatest integer less than or equal to $s$, and denote by $\lceil s\rceil$ the smallest integer greater than or equal to $s$. For a vector $v=\left(v_{1}, \ldots, v_{n}\right)$, denote the sum of all elements in $v$ by $|v|$, i.e., $|v|=v_{1}+\cdots+v_{n}$. Let

$$
v_{n}^{s}=(\underbrace{1,1, \ldots, 1}_{\lfloor s\rfloor}, s-\lfloor s\rfloor, 0, \ldots, 0) \in \mathbb{R}^{n},
$$


which contains $\lfloor s\rfloor$ 's and satisfies $\left|v_{n}^{s}\right|=s$. Denote by $\mathcal{R E}\left(v_{n}^{s}\right)$ the set of all rearrangements of $v_{n}^{s}$, i.e.,

$$
\mathcal{R E}\left(v_{n}^{s}\right)=\left\{v \in \mathbb{R}^{n}: \exists \pi \in S_{n}, \pi(v)=\left(v_{\pi(1)}, v_{\pi(2)}, \ldots, v_{\pi(n)}\right)=v_{n}^{s}\right\} .
$$

For $0 \leq \alpha \leq 1$, let $B_{m}(\alpha)$ be the $m \times m$ matrix in the form:

$$
B_{m}(\alpha)=\left(\begin{array}{cccc}
\alpha & 0 & \cdots & 0 \\
1-\alpha & \alpha & \cdots & 0 \\
\vdots & \ddots & \ddots & \vdots \\
0 & \cdots & 1-\alpha & \alpha
\end{array}\right) .
$$

The extreme points of $\omega_{n}^{s}$ can be characterized as the following proposition.

Proposition 1.3 ([9]). Let $A \in \omega_{n}^{s}$. The fmollowing statements are equivalent:

(a) $A \in \mathfrak{E}\left(\omega_{n}^{s}\right)$.

(b) There exist $R, S \in \mathcal{R E}\left(v_{n}^{s}\right)$, such that $A \in \mathfrak{E}(R, S)$.

(c) There exist $n \times n$ permutation matrices $P$ and $Q$, such that

$$
P A Q=I_{\lceil s\rceil-m} \oplus B_{m}(s+1-\lceil s\rceil) \oplus O_{n-\lceil s\rceil},
$$

for some $1 \leq m \leq\lceil s\rceil$, where $I_{\lceil s\rceil-m}$ is the identity matrix of szize $\lceil s\rceil-m$ and $O_{n-\lceil s\rceil}$ is the zero matrix of size $n-\lceil s\rceil$.

(d) Each connected component of $B G(A)$ is either an isolated vertex or a path. For $s$ not an integer, there exists one path with length $1 \leq m \leq\lceil s\rceil$ and $\lceil s\rceil-m$ paths with length 1 in $B G(A)$. When $s$ is an integer, there are $s$ paths with length 1 in $B G(A)$.

Given nonnegative vectors $\bar{R}=\left(\bar{r}_{1}, \ldots, \bar{r}_{m}\right)$ and $\bar{S}=\left(\bar{s}_{1}, \ldots, \bar{s}_{n}\right)$, consider the set

$$
\Delta(\bar{R}, \bar{S}, \sigma)=\bigcup_{\substack{|R|=|S|=\sigma \\ R \triangleleft \bar{R}, S \triangleleft \bar{S}}} \mathcal{U}(R, S),
$$

where $R \triangleleft \bar{R}$ means $R$ is dominated componentwise by $\bar{R}$, and $S \triangleleft \bar{S}$ means $S$ is dominated componentwise by $\bar{S}$. Here $|\bar{R}|$ is not necessarily equal to $|\bar{S}|$. As we show in the following lemma, a necessary and sufficient condition of $\Delta(\bar{R}, \bar{S}, \sigma)$ being nonempty is simply that $\sigma \leq \min \{|\bar{R}|,|\bar{S}|\}$.

Lemma 1.4. The convex polytope $\Delta(\bar{R}, \bar{S}, \sigma)$ is nonempty if and only if $0 \leq \sigma \leq \min \{|\bar{R}|,|\bar{S}|\}$.

Proof. " $\Leftarrow$ " Without loss of generality, we assume that $\bar{r}_{1} \leq \bar{r}_{2} \leq \cdots \leq \bar{r}_{m}$ and $\bar{s}_{1} \leq \bar{s}_{2} \leq \cdots \leq \bar{s}_{n}$. For any $0 \leq \sigma \leq \min \{|\bar{R}|,|\bar{S}|\}$, there exist some $i_{0}$ and $j_{0}$ such that

$$
\bar{r}_{1}+\cdots+\bar{r}_{i_{0}}<\sigma \leq \bar{r}_{1}+\cdots+\bar{r}_{i_{0}}+\bar{r}_{i_{0}+1}
$$

and

$$
\bar{s}_{1}+\cdots+\bar{s}_{j_{0}}<\sigma \leq \bar{s}_{1}+\cdots+\bar{s}_{j_{0}}+\bar{s}_{j_{0}+1} .
$$

Thus, we can take

$$
\bar{R}_{0}=\left(\bar{r}_{1}, \ldots, \bar{r}_{i_{0}}, \sigma-\sum_{i=1}^{i_{0}} \bar{r}_{i}, 0, \ldots, 0\right)
$$


and

$$
\bar{S}_{0}=\left(\bar{s}_{1}, \ldots, \bar{s}_{j_{0}}, \sigma-\sum_{j=1}^{j_{0}} \bar{s}_{i}, 0, \ldots, 0\right) .
$$

Since $\left|R_{0}\right|=\left|S_{0}\right|=\sigma$ and $R_{0} \triangleleft \bar{R}, S_{0} \triangleleft \bar{S}$, we have $\mathcal{U}\left(R_{0}, S_{0}\right) \subseteq \Delta(\bar{R}, \bar{S}, \sigma)$. Thus $\Delta(\bar{R}, \bar{S}, \sigma)$ is nonempty.

$" \Rightarrow "$ Suppose that $\Delta(\bar{R}, \bar{S}, \sigma) \neq \emptyset$ and $A=\left[a_{i, j}\right] \in \Delta(\bar{R}, \bar{S}, \sigma)$. Since $R(A) \triangleleft \bar{R}, S(A) \triangleleft \bar{S}, \sum_{i, j=1}^{n} a_{i, j}=$ $\sigma$, we have $\sigma=|R(A)| \leq|\bar{R}|$ and $\sigma=|S(A)| \leq|\bar{S}|$. Thus $0 \leq \sigma \leq \min \{|\bar{R}|,|\bar{S}|\}$.

Since $\Delta(\bar{R}, \bar{S}, \sigma)$ is convex, it is natural to ask what are the extreme points of $\Delta(\bar{R}, \bar{S}, \sigma)$. We answer this question in Section 2. In Section 3, we investigate the minimal term rank $\tilde{\rho}$ of the matrices in $\Delta(\bar{R}, \bar{S}, \sigma)$. In Section 4, we study the upper bounds for the permanents of matrices in $\Delta(\bar{R}, \bar{S}, \sigma)$.

2. The extreme points of $\Delta(\bar{R}, \bar{S}, \sigma)$. Given nonnegative vectors

$$
\bar{R}=\left(\bar{r}_{1}, \bar{r}_{2}, \ldots, \bar{r}_{m}\right), \bar{S}=\left(\bar{s}_{1}, \bar{s}_{2}, \ldots, \bar{s}_{n}\right),
$$

and $0 \leq \sigma \leq \min \{|\bar{R}|,|\bar{S}|\}$, denote by $\mathfrak{E}(\Delta(\bar{R}, \bar{S}, \sigma))$ the set of all extreme points of $\Delta(\bar{R}, \bar{S}, \sigma)$. We then characterize the matrices in $\mathfrak{E}(\Delta(\bar{R}, \bar{S}, \sigma))$. We say that the $i$ th row sum (resp. the $j$ th column sum) of a matrix $A$ in $\Delta(\bar{R}, \bar{S}, \sigma)$ is unattained if $0<r_{i}(A)<\bar{r}_{i}$ (resp. $\left.0<s_{j}(A)<\bar{s}_{j}\right)$. Or equivalently, the $i$ th row sum (resp. the $j$ th column sum) vertex in $B G(A)$ is unattained.

THEOREM 2.1. Given nonnegative vectors $\bar{R}=\left(\bar{r}_{1}, \bar{r}_{2}, \ldots, \bar{r}_{m}\right), \bar{S}=\left(\bar{s}_{1}, \bar{s}_{2}, \ldots, \bar{s}_{n}\right)$, and $0 \leq \sigma \leq$ $\min \{|\bar{R}|,|\bar{S}|\}$, if $A \in \mathfrak{E}(\Delta(\bar{R}, \bar{S}, \sigma))$ and $B G(A)$ is connected, then at most one row vertex (resp. one column vertex) corresponding to a row (resp. a column) in $A$ is unattained.

Proof. Assume that both the row vertices $\mathbf{r}_{i}$ and $\mathbf{r}_{j}$ of the connected component corresponding to row $i$ and row $j$, respectively, are unattained. There is a path connecting $\mathbf{r}_{i}$ and $\mathbf{r}_{j}$, i.e.,

$$
\mathbf{r}_{i} \rightarrow \mathbf{s}_{j_{1}} \rightarrow \mathbf{r}_{i_{1}} \rightarrow \mathbf{s}_{j_{2}} \rightarrow \cdots \rightarrow \mathbf{r}_{i_{k-1}} \rightarrow \mathbf{s}_{j_{k}} \rightarrow \mathbf{r}_{j}
$$

We then add an $\epsilon$ to the entries

$$
\left(i, j_{1}\right),\left(i_{1}, j_{2}\right), \ldots,\left(i_{k-1}, j_{k}\right)
$$

and subtract an $\epsilon$ from the entries

$$
\left(i_{1}, j_{1}\right),\left(i_{2}, j_{2}\right), \ldots,\left(i_{k-1}, j_{k-1}\right),\left(j, j_{k}\right)
$$

The resultant matrix is denoted by $A_{1}$. We can illustrate this process by the following path

$$
\mathbf{r}_{i} \stackrel{+\epsilon}{\longrightarrow} \mathbf{s}_{j_{1}} \stackrel{-\epsilon}{\longrightarrow} \mathbf{r}_{i_{1}} \stackrel{+\epsilon}{\longrightarrow} \mathbf{s}_{j_{2}} \stackrel{-\epsilon}{\longrightarrow} \cdots \stackrel{-\epsilon}{\longrightarrow} \mathbf{r}_{i_{k-1}} \stackrel{+\epsilon}{\longrightarrow} \mathbf{s}_{j_{k}} \stackrel{-\epsilon}{\longrightarrow} \mathbf{r}_{j}
$$

Similarly, by reversing the sign of $\epsilon$, we get another matrix $A_{2}$ which can be illustrated by the following path

$$
\mathbf{r}_{i} \longrightarrow \mathbf{s}_{j_{1}} \stackrel{+\epsilon}{\longrightarrow} \mathbf{r}_{i_{1}} \longrightarrow \mathbf{s}_{j_{2}} \stackrel{+\epsilon}{\longrightarrow} \stackrel{+\epsilon}{\longrightarrow} \mathbf{r}_{i_{k-1}} \stackrel{-\epsilon}{\longrightarrow} \mathbf{s}_{j_{k}} \stackrel{+\epsilon}{\longrightarrow} \mathbf{r}_{j}
$$

It is easy to check that

$$
\left|A_{1}\right|=\left|A_{2}\right|=|A|=\sigma,
$$


and

$$
S\left(A_{1}\right)=S\left(A_{2}\right)=S(A)
$$

The row sum vectors of $A_{1}$ and $A_{2}$ differ from $R(A)$ only on the $i$ th and $j$ th row sum entries, which can be shown as

$$
\begin{aligned}
& r_{i}\left(A_{1}\right)=r_{i}(A)+\epsilon, r_{i}\left(A_{2}\right)=r_{i}(A)-\epsilon, \\
& r_{j}\left(A_{1}\right)=r_{j}(A)-\epsilon, r_{j}\left(A_{2}\right)=r_{j}(A)+\epsilon .
\end{aligned}
$$

Since both $r_{i}(A)$ and $r_{j}(A)$ in $R(A)$ are unattained, we can choose $\epsilon$ small enough to make sure that both $A_{1}$ and $A_{2}$ are in $\Delta(\bar{R}, \bar{S}, \sigma)$. Since

$$
A=\frac{1}{2} A_{1}+\frac{1}{2} A_{2}
$$

$A$ is not an extreme point of $\Delta(\bar{R}, \bar{S}, \sigma)$. This contradicts with the given condition. The case that at most one column sum entry in $S(A)$ is unattained can be proved similarly.

REMARK 2.2. When $B G(A)$ contains two or more connected components and $A$ is extreme in $\Delta(\bar{R}, \bar{S}, \sigma)$, by the similar proof as in Theorem 2.1, we conclude that each connected component of $B G(A)$ contains at most one row vertex (resp. one column vertex) which is unattained.

TheOREM 2.3. Given nonnegative vectors $\bar{R}=\left(\bar{r}_{1}, \bar{r}_{2}, \ldots, \bar{r}_{m}\right), \bar{S}=\left(\bar{s}_{1}, \bar{s}_{2}, \ldots, \bar{s}_{n}\right)$, and $0 \leq \sigma \leq$ $\min \{|\bar{R}|,|\bar{S}|\}$, if $A \in \mathfrak{E}(\Delta(\bar{R}, \bar{S}, \sigma))$, then $B G(A)$ contains at most one connected component with exactly one row vertex and one column vertex which are unattained.

Proof. Assume that $B G(A)$ has two connected components, each of which contains exactly one row vertex and one column vertex unattained, implying that $B G(A)$ has two disconnected paths

$$
\mathbf{r}_{i_{1}} \rightarrow \mathbf{s}_{j_{1}} \rightarrow \mathbf{r}_{i_{2}} \rightarrow \mathbf{s}_{j_{2}} \rightarrow \cdots \rightarrow \mathbf{r}_{i_{p}} \rightarrow \mathbf{s}_{j_{p}}
$$

and

$$
\mathbf{r}_{i_{1}^{\prime}} \rightarrow \mathbf{s}_{j_{1}^{\prime}} \rightarrow \mathbf{r}_{i_{2}^{\prime}} \rightarrow \mathbf{s}_{j_{2}^{\prime}} \rightarrow \cdots \rightarrow \mathbf{r}_{i_{q}^{\prime}} \rightarrow \mathbf{s}_{j_{q}^{\prime}}
$$

which are contained in these two connected components, respectively, where $r_{i_{1}}(A)$ and $r_{i_{1}^{\prime}}(A), s_{j_{p}}(A)$ and $s_{j_{q}^{\prime}}(A)$ are unattained. We then add an $\epsilon$ to the entries

$$
\left(i_{1}, j_{1}\right),\left(i_{2}, j_{2}\right), \ldots,\left(i_{p}, j_{p}\right)
$$

and subtract an $\epsilon$ from the entries

$$
\left(i_{2}, j_{1}\right),\left(i_{3}, j_{2}\right), \ldots,\left(i_{p}, j_{p-1}\right) .
$$

The $i_{1}$ th row sum and the $j_{p}$ th column sum are increased by $\epsilon$ while other row and column sums remain unchanged. The total sum of all elements in the connected component containing the path (2.3) is increased by $\epsilon$. We then subtract an $\epsilon$ from the entries

$$
\left(i_{1}^{\prime}, j_{1}^{\prime}\right),\left(i_{2}^{\prime}, j_{2}^{\prime}\right), \ldots,\left(i_{q}^{\prime}, j_{q}^{\prime}\right)
$$


and add an $\epsilon$ to the entries

$$
\left(i_{2}^{\prime}, j_{1}^{\prime}\right),\left(i_{3}^{\prime}, j_{2}^{\prime}\right), \ldots,\left(i_{q}^{\prime}, j_{q-1}^{\prime}\right)
$$

This will decrease the $i_{1}^{\prime}$ th row sum and the $j_{q}^{\prime}$ th column sum by $\epsilon$. The total sum of all elements in the connected component containing the path (2.4) is decreased by $\epsilon$. Denote the resulting matrix by $A^{+}$and note that the total sum of $A^{+}$is still equal to $\sigma$, which remains unchanged. By reversing the sign of $\epsilon$, we obtain another matrix $A^{-}$with the total sum equal to $\sigma$. Since $r_{i_{1}}(A), r_{i_{1}^{\prime}}(A), s_{j_{p}}(A), s_{j_{q}^{\prime}}(A)$ are unattained, we can choose $\epsilon$ sufficiently small such that $A^{+}, A^{-}$are in $\Delta(\bar{R}, \bar{S}, \sigma)$. Since

$$
A=\frac{1}{2} A^{+}+\frac{1}{2} A^{-}
$$

$A$ is not an extreme point in $\Delta(\bar{R}, \bar{S}, \sigma)$. This contradicts with the given condition.

TheOrem 2.4. Let $\bar{R}=\left(\bar{r}_{1}, \ldots, \bar{r}_{m}\right), \bar{S}=\left(\bar{s}_{1}, \ldots, \bar{s}_{n}\right)$ be nonnegative vectors, $0 \leq \sigma \leq \min \{|\bar{R}|,|\bar{S}|\}$, and $A$ is a matrix in $\Delta(\bar{R}, \bar{S}, \sigma)$. A is in $\mathfrak{E}(\Delta(\bar{R}, \bar{S}, \sigma))$ if and only if $B G(A)$ is a forest and BG $(A)$ contains at most one connected component with exactly one unattained row vertex and one unattained column vertex, and all other connected components are formed by the following three types $(i),($ ii $),($ iii $)$,

(i) All row and column sums are attained,

(ii) Exactly one row sum is not attained,

(iii) Exactly one column sum is not attained.

Proof. " $\Rightarrow$ " By the definition of $\Delta(\bar{R}, \bar{S}, \sigma)$ in (1.2), we have

$$
\mathfrak{E}(\Delta(\bar{R}, \bar{S}, \sigma)) \subseteq \bigcup_{\substack{|R|=|S|=\sigma \\ R \triangleleft \bar{R}, S \triangleleft \bar{S}}} \mathfrak{E}(R, S)
$$

where $\mathfrak{E}(R, S)$ denotes the set of all extreme points of $\mathcal{U}(R, S)$. If $A \in \mathfrak{E}(\Delta(\bar{R}, \bar{S}, \sigma))$, then there exist $R \triangleleft \bar{R}$ and $S \triangleleft \bar{S}$ with $|R|=|S|=\sigma$ such that $A \in \mathfrak{E}(R, S)$. By Proposition 1.1, BG(A) is a forest. Theorem 2.1 guarantees that the connected components of $B G(A)$ are of types $(i),($ ii $),($ iii $)$, and Theorem 2.3 guarantees that $B G(A)$ contains at most one connected component with exactly one unattained row vertex and one unattained column vertex.

" $\Leftarrow "$ Let $A$ be a matrix in $\Delta(\bar{R}, \bar{S}, \sigma)$ and $B G(A)$ satisfy the given conditions. Suppose that there exist $A_{1}$ and $A_{2}$ both in $\Delta(\bar{R}, \bar{S}, \sigma)$ such that

$$
A=\lambda A_{1}+(1-\lambda) A_{2}
$$

for some $0<\lambda<1$. By Proposition 1.1, $A \in \mathfrak{E}(R(A), S(A))$ where $|R(A)|=|S(A)|=\sigma$ and $R(A) \triangleleft$ $\bar{R}, S(A) \triangleleft \bar{S}$. Since the bipartite graphs $B G\left(A_{1}\right)$ and $B G\left(A_{2}\right)$ are subgraphs of $B G(A)$, both $B G\left(A_{1}\right)$ and $B G\left(A_{2}\right)$ are forests. Therefore, $A_{1} \in \mathfrak{E}\left(R\left(A_{1}\right), S\left(A_{1}\right)\right)$ and $A_{2} \in \mathfrak{E}\left(R\left(A_{2}\right), S\left(A_{2}\right)\right)$, where $\left|R\left(A_{1}\right)\right|=$ $\left|S\left(A_{1}\right)\right|=\left|R\left(A_{2}\right)\right|=\left|S\left(A_{2}\right)\right|=\sigma$ and $R\left(A_{1}\right) \triangleleft \bar{R}, S\left(A_{1}\right) \triangleleft \bar{S}, R\left(A_{2}\right) \triangleleft \bar{R}, S\left(A_{2}\right) \triangleleft \bar{S}$. Moreover, if $r_{i}(A)$ (resp. $\left.s_{j}(A)\right)$ is attained, by $(2.5)$ both $r_{i}\left(A_{1}\right)$ (resp. $s_{j}\left(A_{1}\right)$ ) and $r_{i}\left(A_{2}\right)$ (resp. $s_{j}\left(A_{2}\right)$ ) are attained. Since the connected component of $B G(A)$ which is of type $(i),(i i)$, or $(i i i)$ contains at most one unattained vertex, $B G\left(A_{1}\right)$ and $B G\left(A_{2}\right)$ must have exactly the same connected components as those in $B G(A)$ which are of types $(i),(i i),(i i i)$. If $B G(A)$ contains at most one connected component with exactly one unattained row vertex and one unattained column vertex, then in this connected component, the only unattained row sum and the only unattained column sum are uniquely determined since the rest row and column sums are all 
attained. Therefore, we claim that $R\left(A_{1}\right)=R\left(A_{2}\right)=R(A), S\left(A_{1}\right)=S\left(A_{2}\right)=S(A)$. This implies that $A, A_{1}, A_{2} \in \mathfrak{E}(R(A), S(A))$ and $A=A_{1}=A_{2}$. Thus, $A$ is extreme in $\Delta(\bar{R}, \bar{S}, \sigma)$.

CoROllary 2.5. If $\bar{R}=\left(\bar{r}_{1}, \ldots, \bar{r}_{m}\right), \bar{S}=\left(\bar{s}_{1}, \ldots, \bar{s}_{n}\right)$ are nonnegative vectors with $|\bar{R}|=|\bar{S}|$ and $0 \leq \sigma \leq|\bar{R}|$, then

$$
\left\{B \in \mathfrak{E}_{\leq}(\bar{R}, \bar{S})|| B \mid=\sigma\right\} \subseteq \mathfrak{E}(\Delta(\bar{R}, \bar{S}, \sigma)) .
$$

Proof. This follows from the fact that $\Delta(\bar{R}, \bar{S}, \sigma)=\left\{A \in \mathcal{U}_{\leq}(\bar{R}, \bar{S})|| A \mid=\sigma\right\} \subseteq \mathcal{U}_{\leq}(\bar{R}, \bar{S})$ and $\{B \in$ $\left.\mathfrak{E}_{\leq}(\bar{R}, \bar{S})|| B \mid=\sigma\right\} \subseteq \Delta(\bar{R}, \bar{S}, \sigma)$.

Corollary 2.6. Let $\bar{R}=\left(\bar{r}_{1}, \ldots, \bar{r}_{m}\right), \bar{S}=\left(\bar{s}_{1}, \ldots, \bar{s}_{n}\right)$ be nonnegative vectors satisfying that $|\bar{R}|=|\bar{S}|$, $0 \leq \sigma<|\bar{R}|$, and $B$ is extreme in $\mathcal{U}_{\leq}(\bar{R}, \bar{S})$ with $|B|>\sigma$. If there exists one edge connecting $\boldsymbol{r}_{i}$ and $\boldsymbol{s}_{j}$ in $B G(B)$, such that either $\boldsymbol{r}_{i}$ or $\boldsymbol{s}_{j}$ is the only possible unattained vertex in its connected component, and $b_{i, j} \geq|B|-\sigma$, then the matrix obtained from $B$ by decreasing $b_{i, j}$ by $|B|-\sigma$ is an extreme point of $\Delta(\bar{R}, \bar{S}, \sigma)$.

Proof. From Proposition 1.2, $B G(B)$ is a forest and each connected component in $B G(B)$ contains at most one vertex unattained. By decreasing the element $b_{i, j}, B G(B)$ contains at most one connected component with exactly one unattained row vertex and one unattained column vertex. Thus by Theorem 2.4, the matrix obtained from $B$ by decreasing $b_{i, j}$ by $|B|-\sigma$ is an extreme point in $\Delta(\bar{R}, \bar{S}, \sigma)$.

However, $\Delta(\bar{R}, \bar{S}, \sigma)$ contains more extreme points than those in Corollary 2.6. To explicitly obtain the matrices in $\mathfrak{E}(\Delta(\bar{R}, \bar{S}, \sigma))$, we first find out all possible row sum vectors and column sum vectors for matrices in $\mathfrak{E}(\Delta(\bar{R}, \bar{S}, \sigma))$. To do that, we introduce some notations.

Denote the set $\left\{\bar{r}_{1}, \ldots, \bar{r}_{m}\right\}$ by $S_{\bar{R}}$, and the set $\left\{\bar{s}_{1}, \ldots, \bar{s}_{n}\right\}$ by $S_{\bar{S}}$. Let

$$
\bar{R}_{\Sigma}:=\left\{\sum_{r \in W} r \mid W \subseteq S_{\bar{R}}\right\}, \bar{S}_{\Sigma}:=\left\{\sum_{s \in T} s \mid T \subseteq S_{\bar{S}}\right\} .
$$

If $T$ or $W$ is $\emptyset$, then $\sum_{r \in \emptyset} r:=0, \sum_{s \in \emptyset} s:=0$. Also let

$$
\begin{aligned}
& \operatorname{Diff}\left(\bar{R}_{\sum}, \bar{S}_{\sum}\right):=\left\{r-s \mid r-s \geq 0, r \in \bar{R}_{\sum}, s \in \bar{S}_{\sum}\right\}, \\
& \operatorname{Diff}\left(\bar{S}_{\sum}, \bar{R}_{\sum}\right):=\left\{s-r \mid s-r \geq 0, r \in \bar{R}_{\sum}, s \in \bar{S}_{\sum}\right\}, \\
& \operatorname{Sum}\left(\bar{R}_{\sum}, \bar{S}_{\sum}\right):=\left\{r+s \mid r \in \bar{R}_{\sum}, s \in \bar{S}_{\sum}\right\}, \\
& \operatorname{Diff}\left(\sigma, \operatorname{Sum}\left(\bar{R}_{\sum}, \bar{S}_{\sum}\right)\right):=\left\{\sigma-t \mid \sigma-t \geq 0, t \in \operatorname{Sum}\left(\bar{R}_{\sum}, \bar{S}_{\sum}\right)\right\} .
\end{aligned}
$$

Since $0 \in \bar{R}_{\sum}$ and also $0 \in \bar{S}_{\sum}, \bar{R}_{\sum} \subseteq \operatorname{Diff}\left(\bar{R}_{\sum}, \bar{S}_{\sum}\right)$ and $\bar{S}_{\sum} \subseteq \operatorname{Diff}\left(\bar{S}_{\sum}, \bar{R}_{\sum}\right)$.

Define

$$
\mathbf{R}:=\left\{r \leq \sigma: r \in S_{\bar{R}} \cup \operatorname{Diff}\left(\bar{S}_{\sum}, \bar{R}_{\sum}\right) \cup \operatorname{Diff}\left(\sigma, \operatorname{Sum}\left(\bar{R}_{\sum}, \bar{S}_{\sum}\right)\right)\right\}
$$

and

$$
\mathbf{S}:=\left\{s \leq \sigma: s \in S_{\bar{S}} \cup \operatorname{Diff}\left(\bar{R}_{\sum}, \bar{S}_{\sum}\right) \cup \operatorname{Diff}\left(\sigma, \operatorname{Sum}\left(\bar{R}_{\sum}, \bar{S}_{\sum}\right)\right)\right\}
$$

Lemma 2.7. If $\bar{R}=\left(\bar{r}_{1}, \ldots, \bar{r}_{m}\right), \bar{S}=\left(\bar{s}_{1}, \ldots, \bar{s}_{n}\right)$ are nonnegative vectors with $0 \leq \sigma \leq \min \{|\bar{R}|,|\bar{S}|\}$, and $A \in \mathfrak{E}(\Delta(\bar{R}, \bar{S}, \sigma))$, then all row sums of $A$ are in the set $\mathbf{R}$ and all column sums of $A$ are in the set $\mathbf{S}$. 
Proof. By Theorem 2.4, $B G(A)$ contains at most one connected component with exactly one unattained row vertex and one unattained column vertex, and all other connected components are of three types (i), (ii) and (iii). If the connected component is of type (i) or type (iii), then the corresponding row sums are all attained which are in $S_{\bar{R}}$. If the connected component is of type (ii), then the unattained row sum is in $\operatorname{Diff}\left(\bar{S}_{\sum}, \bar{R}_{\sum}\right)$ due to the compatibility of the connected component. If the connected component has exactly one unattained row vertex and one unattained column vertex, then the only unattained row sum can then be calculated by taking the difference of $\sigma$ and the rest row sums, which is in $\operatorname{Diff}\left(\sigma, \operatorname{Sum}\left(\bar{S}_{\sum}, \bar{R}_{\sum}\right)\right)$. Since this type of component can appear in $B G(A)$ at most once, $R(A)$ should contain at most one element which is generated in this way. The column sum case can be proved similarly.

From Theorem 2.4, we have the following proposition and corollary.

Proposition 2.8. Given nonnegative vectors $\bar{R}=\left(\bar{r}_{1}, \ldots, \bar{r}_{m}\right), \bar{S}=\left(\bar{s}_{1}, \ldots, \bar{s}_{n}\right)$, and $0 \leq \sigma \leq$ $\min \{|\bar{R}|,|\bar{S}|\}$, if $A \in \mathfrak{E}(\Delta(\bar{R}, \bar{S}, \sigma))$, then the number of unattained line sums in $A$ does not exceed the number of connected components in $B G(A)$ plus one.

Corollary 2.9. Given nonnegative vectors $\bar{R}=\left(\bar{r}_{1}, \ldots, \bar{r}_{m}\right), \bar{S}=\left(\bar{s}_{1}, \ldots, \bar{s}_{n}\right)$, and $0 \leq \sigma \leq$ $\min \{|\bar{R}|,|\bar{S}|\}$, if $A \in \mathfrak{E}(\Delta(\bar{R}, \bar{S}, \sigma))$, then the number of unattained line sums in $A$ is at most $\min \{m, n\}+1$.

Proof. Since in $B G(A)$ the number of connected components containing at least one edge is at most $\min \{m, n\}$, together with Theorem 2.4, there are at most $\min \{m, n\}+1$ unattained vertices.

Corollary 2.9 helps us to exclude those pairs $(\tilde{R}, \tilde{S})$ whose corresponding transportation polytopes do not contain any extreme points of $\Delta(\bar{R}, \bar{S}, \sigma)$, and we have

$$
\mathfrak{E}(\Delta(\bar{R}, \bar{S}, \sigma)) \subseteq \bigcup_{\substack{|\tilde{R}|=|\tilde{S}|=\sigma \\ \tilde{R} \triangleleft \bar{R}, \bar{S} \triangleleft \bar{S}}} \mathfrak{E}(\tilde{R}, \tilde{S}),
$$

where $\tilde{R}, \tilde{S}$ satisfy the conditions in Lemma 2.7 and Corollary 2.9. When $\sigma<\min \{|\bar{R}|,|\bar{S}|\}, \mathfrak{E}(\Delta(\bar{R}, \bar{S}, \sigma))$ is a proper subset of $\bigcup \mathfrak{E}(\tilde{R}, \tilde{S})$, where $|\tilde{R}|=|\tilde{S}|=\sigma$ and $\tilde{R} \triangleleft \bar{R}, \tilde{S} \triangleleft \bar{S}$. This is because not all matrices in $\mathfrak{E}(\tilde{R}, \tilde{S})$ satisfy Theorem 2.4. In order to obtain the matrices in $\mathfrak{E}(\Delta(\bar{R}, \bar{S}, \sigma))$, we first find out all extreme points of $\mathcal{U}(\tilde{R}, \tilde{S})$ as long as $\tilde{R}$ and $\tilde{S}$ satisfy the conditions in Lemma 2.7 and Corollary 2.9. Then remove those matrices in $\mathfrak{E}(\tilde{R}, \tilde{S})$ whose bipartite graphs do not satisfy the conditions in Theorem 2.4.

Here, we use an example to illustrate how to find the right pairs of $\tilde{R}$ and $\tilde{S}$.

ExAmple 2.10. Given $\sigma=4, \bar{R}=(2,3), \bar{S}=(1,2,3)$, we have $S_{\bar{R}}=\{2,3\}, S_{\bar{S}}=\{1,2,3\}$ and

$$
\begin{aligned}
& \bar{R}_{\Sigma}=\{0,2,3,5\}, \bar{S}_{\Sigma}=\{0,1,2,3,4,5,6\}, \\
& \operatorname{Diff}\left(\bar{R}_{\sum}, \bar{S}_{\sum}\right)=\{0,1,2,3,4,5\}, \\
& \operatorname{Diff}\left(\bar{S}_{\sum}, \bar{R}_{\sum}\right)=\{0,1,2,3,4,5,6\}, \\
& \operatorname{Sum}\left(\bar{R}_{\sum}, \bar{S}_{\sum}\right)=\{0,1,2,3,4,5,6,7,8,9,10,11\}, \\
& \operatorname{Diff}\left(\sigma, \operatorname{Sum}\left(\bar{R}_{\sum}, \bar{S}_{\sum}\right)\right)=\{0,1,2,3,4\}, \\
& \mathbf{R}=\mathbf{S}=\{0,1,2,3,4\} .
\end{aligned}
$$

The row and column sum vectors of the polytopes satisfying Corollary 2.9 are as follows.

1. $\tilde{R}_{1}=(1,3), \tilde{S}_{1}=(0,1,3)$;

2. $\tilde{R}_{2}=(1,3), \tilde{S}_{2}=(0,2,2)$; 
3. $\tilde{R}_{3}=(1,3), \tilde{S}_{3}=(1,0,3)$;

4. $\tilde{R}_{4}=(1,3), \tilde{S}_{4}=(1,1,2)$;

5. $\tilde{R}_{5}=(1,3), \tilde{S}_{5}=(1,2,1)$;

6. $\tilde{R}_{6}=(2,2), \tilde{S}_{6}=(0,1,3)$;

7. $\tilde{R}_{7}=(2,2), \tilde{S}_{7}=(0,2,2)$;

8. $\tilde{R}_{8}=(2,2), \tilde{S}_{8}=(1,0,3)$;

9. $\tilde{R}_{9}=(2,2), \tilde{S}_{9}=(1,1,2)$;

10. $\tilde{R}_{10}=(2,2), \tilde{S}_{10}=(1,2,1)$.

Once we have all possible $\tilde{R}$ and $\tilde{S}$, we can also modify the Ryser's algorithm ([5, 17]) to obtain the extreme points of $\Delta(\bar{R}, \bar{S}, \sigma)$.

(i) Let $\tilde{R}=\left(\tilde{r}_{1}, \ldots, \tilde{r}_{m}\right)$ and $\tilde{S}=\left(\tilde{s}_{1}, \ldots, \tilde{s}_{n}\right)$ where $\tilde{r}_{1}+\cdots+\tilde{r}_{m}=\tilde{s}_{1}+\cdots+\tilde{s}_{n}=\sigma$. Begin with $\hat{R}=\tilde{R}$ and $\hat{S}=\tilde{S}$, and $A=\left[a_{i, j}\right]$ equal to zero matrix of size $m \times n$.

(ii) Choose $\tilde{r}_{i}$ and $\tilde{s}_{j}$ and replace $a_{i, j}$ with $\min \left\{\tilde{r}_{i}, \tilde{s}_{j}\right\}$. Let

$$
\hat{R}=\left(\tilde{r}_{1}, \ldots, \tilde{r}_{i}-\min \left\{\tilde{r}_{i}, \tilde{s}_{j}\right\}, \ldots, \tilde{r}_{m}\right),
$$

and

$$
\hat{S}=\left(\tilde{s}_{1}, \ldots, \tilde{s}_{j}-\min \left\{\tilde{r}_{i}, \tilde{s}_{j}\right\}, \ldots, \tilde{s}_{n}\right) .
$$

If $\tilde{r}_{i}<\bar{r}_{i}$ unattained, then all the row vertices in the same connected component containing row vertex $\mathbf{r}_{i}$ must be attained. If $\tilde{s}_{j}<\bar{s}_{j}$ unattained, then all the column vertices in the same connected component containing column vertex $\mathbf{s}_{j}$ must be attained. Once there is a connected component in $B G(A)$ which contains exactly one row vertex and one column vertex which are both unattained, then all the other connected components should only contain at most one unattained vertex. Otherwise, we stop the procedure and output empty.

(iii) Repeat Step(ii) until $\hat{R}$ and $\hat{S}$ are zero vectors.

EXAmple 2.11. Following the results of Example 2.10, we can find all the extreme points of $\Delta(\bar{R}, \bar{S}, \sigma)$ where $\sigma=4, \bar{R}=(2,3), \bar{S}=(1,2,3)$ as listed below

$$
\begin{aligned}
& {\left[\begin{array}{lll}
0 & 1 & 0 \\
0 & 0 & 3
\end{array}\right],\left[\begin{array}{lll}
0 & 0 & 1 \\
0 & 1 & 2
\end{array}\right],\left[\begin{array}{lll}
0 & 1 & 0 \\
0 & 1 & 2
\end{array}\right],\left[\begin{array}{lll}
0 & 0 & 1 \\
0 & 2 & 1
\end{array}\right],\left[\begin{array}{lll}
1 & 0 & 0 \\
0 & 0 & 3
\end{array}\right],\left[\begin{array}{lll}
0 & 0 & 1 \\
1 & 0 & 2
\end{array}\right],} \\
& {\left[\begin{array}{lll}
0 & 1 & 0 \\
1 & 0 & 2
\end{array}\right],\left[\begin{array}{lll}
1 & 0 & 0 \\
0 & 2 & 1
\end{array}\right],\left[\begin{array}{lll}
0 & 1 & 0 \\
1 & 1 & 1
\end{array}\right],\left[\begin{array}{lll}
0 & 0 & 1 \\
1 & 2 & 0
\end{array}\right],\left[\begin{array}{lll}
0 & 1 & 1 \\
0 & 0 & 2
\end{array}\right],\left[\begin{array}{lll}
0 & 0 & 2 \\
0 & 1 & 1
\end{array}\right],} \\
& {\left[\begin{array}{lll}
0 & 2 & 0 \\
0 & 0 & 2
\end{array}\right],\left[\begin{array}{lll}
0 & 0 & 2 \\
0 & 2 & 0
\end{array}\right],\left[\begin{array}{lll}
1 & 0 & 1 \\
0 & 0 & 2
\end{array}\right],\left[\begin{array}{lll}
0 & 0 & 2 \\
1 & 0 & 1
\end{array}\right],\left[\begin{array}{lll}
1 & 1 & 0 \\
0 & 0 & 2
\end{array}\right],\left[\begin{array}{lll}
0 & 0 & 2 \\
1 & 1 & 0
\end{array}\right],} \\
& {\left[\begin{array}{lll}
1 & 0 & 1 \\
0 & 2 & 0
\end{array}\right],\left[\begin{array}{lll}
1 & 1 & 0 \\
0 & 1 & 1
\end{array}\right],\left[\begin{array}{lll}
0 & 1 & 1 \\
1 & 1 & 0
\end{array}\right],\left[\begin{array}{lll}
0 & 2 & 0 \\
1 & 0 & 1
\end{array}\right],}
\end{aligned}
$$

Remark 2.12. Denote $L_{n}=(1,1, \ldots, 1) \in \mathbb{R}^{n}$. Note that $\omega_{n}^{s}=\Delta\left(L_{n}, L_{n}, s\right)$ where $0 \leq s \leq n$. By Lemma 2.7, $\mathbf{R}=\mathbf{S}=\{0,1, s-\lfloor s\rfloor\}$. Therefore, Proposition 1.3 easily follows from Theorem 2.4.

3. The minimal term rank. Let $A=\left[a_{i, j}\right]$ be an $m \times n$ nonnegative matrix. The term rank of $A$ is the maximal number $\rho(A)$ of positive elements of $A$ with no two of them on a line. The fundamental 
Electronic Journal of Linear Algebra, ISSN 1081-3810

A publication of the International Linear Algebra Society

Volume 37, pp. 256-271, March 2021.

minimax theorem of König-Egerváry asserts that $\rho(A)$ equals the minimal number of lines in $A$ that contain all positive elements in $A[5]$. In this section, we investigate the minimal term rank $\tilde{\rho}$ of the matrices in the class $\Delta(\bar{R}, \bar{S}, \sigma)$. Given set $S$, denote by $\tilde{\rho}(S)$ the minimal term rank of the matrices in $S$. We first show that the minimal term rank can only be achieved on the extreme points of $\Delta(\bar{R}, \bar{S}, \sigma)$.

LEMMA 3.1. Let $S$ be a bounded convex polytope of $m \times n$ matrices, and $\mathfrak{E}(S)$ be the set of all extreme points of $S$ which is nonempty. Then

$$
\tilde{\rho}(S)=\tilde{\rho}(\mathfrak{E}(S))
$$

Proof. Since $\mathfrak{E}(S) \subseteq S, \tilde{\rho}(S) \leq \tilde{\rho}(\mathfrak{E}(S))$. On the other hand, for any $A \in S$, there exist $A_{1}, \ldots, A_{k} \in \mathfrak{E}(S)$ such that

$$
A=\lambda_{1} A_{1}+\cdots+\lambda_{k} A_{k}
$$

where $0 \leq \lambda_{1}, \ldots, \lambda_{k} \leq 1$ and $\lambda_{1}+\cdots+\lambda_{k}=1$. Thus, a cover of $A$ is also a cover of $A_{i}$ for $1 \leq i \leq k$. By the minimax theorem this implies that $\rho(A) \geq \rho\left(A_{i}\right)$ for $1 \leq i \leq k$, and $\tilde{\rho}(S) \geq \tilde{\rho}(\mathfrak{E}(S))$. Therefore, the lemma holds.

THEOREM 3.2. Let $\bar{R}=\left(\bar{r}_{1}, \ldots, \bar{r}_{m}\right)$ and $\bar{S}=\left(\bar{s}_{1}, \ldots, \bar{s}_{n}\right)$ be nonnegative vectors, and $0 \leq \sigma \leq$ $\min \{|\bar{R}|,|\bar{S}|\}$. We have

$$
\tilde{\rho}(\Delta(\bar{R}, \bar{S}, \sigma))=\min \{\tilde{\rho}(\mathfrak{E}(\tilde{R}, \tilde{S})):|\tilde{R}|=|\tilde{S}|=\sigma, \tilde{R} \triangleleft \bar{R}, \tilde{S} \triangleleft \bar{S}\},
$$

where $\tilde{R}, \tilde{S}$ satisfy the conditions in Lemma 2.7 and Corollary 2.9.

Proof. Recall that (2.6) says

$$
\left.\mathfrak{E}(\Delta(\bar{R}, \bar{S}, \sigma)) \subseteq \bigcup_{\substack{|\tilde{R}|=|\tilde{S}|=\sigma \\ \tilde{R} \triangleleft \bar{R}, \tilde{S} \triangleleft \bar{S}}} \mathfrak{E}(\tilde{R}, \tilde{S}) \subseteq \Delta(\bar{R}, \bar{S}, \sigma)\right)
$$

where $\tilde{R}, \tilde{S}$ satisfy the conditions in Lemma 2.7 and Corollary 2.9. Therefore,

$$
\tilde{\rho}(\mathfrak{E}(\Delta(\bar{R}, \bar{S}, \sigma))) \geq \min \{\tilde{\rho}(\mathfrak{E}(\tilde{R}, \tilde{S})):|\tilde{R}|=|\tilde{S}|=\sigma, \tilde{R} \triangleleft \bar{R}, \tilde{S} \triangleleft \bar{S}\} \geq \tilde{\rho}(\Delta(\bar{R}, \bar{S}, \sigma))
$$

By Lemma 3.1, $\tilde{\rho}(\Delta(\bar{R}, \bar{S}, \sigma))=\tilde{\rho}(\mathfrak{E}(\Delta(\bar{R}, \bar{S}, \sigma)))$. Therefore the theorem holds.

For any nonnegative real vector $R=\left(a_{1}, a_{2}, \ldots, a_{l}\right)$, denote the rearrangement of the elements in $R$ in ascending order by

$$
a_{1}^{\prime} \leq a_{2}^{\prime} \leq \cdots \leq a_{l}^{\prime}
$$

and in descending order by

$$
a_{1}^{*} \geq a_{2}^{*} \geq \cdots \geq a_{l}^{*}
$$

We denote

$$
R^{\prime}=\left(a_{1}^{\prime}, a_{2}^{\prime}, \ldots, a_{l}^{\prime}\right), R^{*}=\left(a_{1}^{*}, a_{2}^{*}, \ldots, a_{l}^{*}\right) .
$$

The following theorem comes from Jurkat and Ryser [17]. 
THEOREM $3.3([17])$. Let $R=\left(r_{1}, \ldots, r_{m}\right)$ and $S=\left(s_{1}, \ldots, s_{n}\right)$ be nonnegative vectors satisfying $|R|=|S|=\sigma$. Then

$$
\tilde{\rho}(\mathcal{U}(R, S))=\rho\left(E_{R^{\prime}, S^{*}}\right),
$$

where $E_{R^{\prime}, S^{*}} \in \mathfrak{E}\left(R^{\prime}, S^{*}\right)$ is constructed inductively by choosing the position $(1,1)$ in the submatrix under consideration. Moreover,

$$
\rho\left(E_{R^{\prime}, S^{*}}\right)=\min \{e+f\}
$$

where the minimum is taken over all pairs $e \geq 0, f \geq 0$ satisfying

$$
r_{1}^{*}+r_{2}^{*}+\cdots+r_{e}^{*}+s_{1}^{*}+s_{2}^{*}+\cdots+s_{f}^{*} \geq \sigma
$$

Given $\bar{R}, \bar{S}$ nonnegative vectors and $0 \leq \sigma \leq \min \{|\bar{R}|,|\bar{S}|\}$, there exist $i_{\sigma}$ and $j_{\sigma}$ such that

$$
\bar{r}_{1}^{*}+\bar{r}_{2}^{*}+\cdots+\bar{r}_{i_{\sigma}-1}^{*}<\sigma \leq \bar{r}_{1}^{*}+\bar{r}_{2}^{*}+\cdots+\bar{r}_{i_{\sigma}}^{*}
$$

and

$$
\bar{s}_{1}^{*}+\bar{s}_{2}^{*}+\cdots+\bar{s}_{j_{\sigma}-1}^{*}<\sigma \leq \bar{s}_{1}^{*}+\bar{s}_{2}^{*}+\cdots+\bar{s}_{j_{\sigma}}^{*}
$$

Let

$$
\begin{aligned}
& R_{\rho}^{*}:=\left(\bar{r}_{1}^{*}, \ldots, \bar{r}_{i_{\sigma}-1}^{*}, \sigma-\sum_{s=1}^{i_{\sigma}-1} \bar{r}_{s}^{*}, 0, \ldots, 0\right), \\
& S_{\rho}^{*}:=\left(\bar{s}_{1}^{*}, \ldots, \bar{s}_{j_{\sigma}-1}^{*}, \sigma-\sum_{t=1}^{j_{\sigma}-1} \bar{s}_{t}^{*}, 0, \ldots, 0\right) .
\end{aligned}
$$

Since $0<\sigma-\sum_{s=1}^{i_{\sigma}-1} \bar{r}_{s}^{*} \leq \bar{r}_{i_{\sigma}}^{*}, 0<\sigma-\sum_{t=1}^{j_{\sigma}-1} \bar{s}_{t}^{*} \leq \bar{s}_{j_{\sigma}}^{*}$, the only possible unattained row sum in $R_{\rho}^{*}$ is the $i_{\sigma}$ th row sum, and the only possible unattained column sum in $S_{\rho}^{*}$ is the $j_{\sigma}$ th column sum. By Theorem 2.4

$$
\mathfrak{E}\left(R_{\rho}^{*}, S_{\rho}^{*}\right) \subseteq \mathfrak{E}(\Delta(\bar{R}, \bar{S}, \sigma))
$$

which implies that

$$
\tilde{\rho}\left(\mathcal{U}\left(R_{\rho}^{*}, S_{\rho}^{*}\right)\right) \geq \tilde{\rho}(\Delta(\bar{R}, \bar{S}, \sigma)) .
$$

On the other hand, for any $\tilde{R}, \tilde{S}$ satisfying $\tilde{R} \triangleleft \bar{R}, \tilde{S} \triangleleft \bar{S}$, and $|\tilde{R}|=|\tilde{S}|=\sigma$, by Theorem 3.3 we have

$$
\tilde{\rho}(\mathcal{U}(\tilde{R}, \tilde{S})) \geq \tilde{\rho}\left(\mathcal{U}\left(R_{\rho}^{*}, S_{\rho}^{*}\right)\right)
$$

(3.7) and (3.8) together give us the following theorem.

THEOREM 3.4. Let $\bar{R}=\left(\bar{r}_{1}, \ldots, \bar{r}_{m}\right)$ and $\bar{S}=\left(\bar{s}_{1}, \ldots, \bar{s}_{n}\right)$ be nonnegative vectors, and $0 \leq \sigma \leq$ $\min \{|\bar{R}|,|\bar{S}|\}$. Then

$$
\tilde{\rho}(\Delta(\bar{R}, \bar{S}, \sigma))=\tilde{\rho}\left(\mathfrak{E}\left(R_{\rho}^{*}, S_{\rho}^{*}\right)\right) .
$$

Corollary 3.5. For $0 \leq s \leq n$, we have

$$
\tilde{\rho}\left(\omega_{n}^{s}\right)=\tilde{\rho}\left(\Delta\left(L_{n}, L_{n}, s\right)\right)=\tilde{\rho}\left(\mathfrak{E}\left(v_{n}^{s}, v_{n}^{s}\right)\right)=\lceil s\rceil .
$$


Electronic Journal of Linear Algebra, ISSN 1081-3810

4. The maximal permanent. In this section, we consider $\Delta(\bar{R}, \bar{S}, \sigma)$ where $\bar{R}$ and $\bar{S}$ are both $n$ dimensional nonnegative real vectors. For an $n \times n$ matrix $A$, the permanent of $A$ is defined by

$$
\operatorname{per}(A)=\sum_{\pi \in S_{n}} a_{1, \pi(1)} a_{2, \pi(2)} \cdots a_{n, \pi(n)},
$$

where $S_{n}$ is the symmetric group of size $n$. The following theorem is given by Jurkat and Ryser [17].

TheOREM 4.1 ([17]). Let $A$ be a matrix in $\mathcal{U}(R, S)$, then

$$
\operatorname{per}(A) \leq \prod_{i=1}^{n} \min \left\{r_{i}^{\prime}, s_{i}^{\prime}\right\}
$$

and equality is attained in (4.9) by a matrix $A$ in $\mathfrak{E}\left(R^{\prime}, S^{\prime}\right)$ with the main diagonal

$$
a_{i, i}=\min \left\{r_{i}^{\prime}, s_{i}^{\prime}\right\}, \text { for all } 1 \leq i \leq n
$$

We then have the following lemma.

LEMMA 4.2. Given nonnegative vectors $\bar{R}=\left(\bar{r}_{1}, \ldots, \bar{r}_{n}\right), \bar{S}=\left(\bar{s}_{1}, \ldots, \bar{s}_{n}\right)$, and $0 \leq \sigma \leq \min \{|\bar{R}|,|\bar{S}|\}$, let $A$ be a matrix in $\Delta(\bar{R}, \bar{S}, \sigma)$. Then

$$
\operatorname{per}(A) \leq \prod_{i=1}^{n} \min \left\{\bar{r}_{i}^{\prime}, \bar{s}_{i}^{\prime}\right\}
$$

Moreover, when $\sigma \geq \sum_{i=1}^{n} \min \left\{\bar{r}_{i}^{\prime}, \bar{s}_{i}^{\prime}\right\}$, there exist matrices with the main diagonal $a_{i, i}=\min \left\{\bar{r}_{i}^{\prime}, \bar{s}_{i}^{\prime}\right\}$ for all $1 \leq i \leq n$ which make (4.10) hold as an equality.

Proof. Recall that

$$
\Delta(\bar{R}, \bar{S}, \sigma)=\bigcup_{\substack{|R|=|S|=\sigma \\ R \triangleleft \bar{R}, S \triangleleft \bar{S}}} \mathcal{U}(R, S) .
$$

For any $R, S$ satisfying $R \triangleleft \bar{R}$, and $S \triangleleft \bar{S},|R|=|S|=\sigma$, we have

$$
r_{1}^{\prime} \leq \bar{r}_{1}^{\prime}, r_{2}^{\prime} \leq \bar{r}_{2}^{\prime}, \ldots, r_{n}^{\prime} \leq \bar{r}_{n}^{\prime}, \quad s_{1}^{\prime} \leq \bar{s}_{1}^{\prime}, s_{2}^{\prime} \leq \bar{s}_{2}^{\prime}, \ldots, s_{n}^{\prime} \leq \bar{s}_{n}^{\prime}
$$

Therefore, $\mathcal{U}\left(R^{\prime}, S^{\prime}\right) \subseteq \Delta\left(\bar{R}^{\prime}, \bar{S}^{\prime}, \sigma\right)$ and for all $1 \leq i \leq n$ we have $\min \left\{r_{i}^{\prime}, s_{i}^{\prime}\right\} \leq \min \left\{\bar{r}_{i}^{\prime}, \bar{s}_{i}^{\prime}\right\}$. This implies that

$$
\prod_{i=1}^{n} \min \left\{r_{i}^{\prime}, s_{i}^{\prime}\right\} \leq \prod_{i=1}^{n} \min \left\{\bar{r}_{i}^{\prime}, \bar{s}_{i}^{\prime}\right\} .
$$

Moreover, by Theorem 4.1, for any $A \in \mathcal{U}(R, S)$,

$$
\operatorname{per}(A) \leq \prod_{i=1}^{n} \min \left\{r_{i}^{\prime}, s_{i}^{\prime}\right\}
$$

Thus for all $A \in \Delta(\bar{R}, \bar{S}, \sigma)$,

$$
\operatorname{per}(A) \leq \prod_{i=1}^{n} \min \left\{\bar{r}_{i}^{\prime}, \bar{s}_{i}^{\prime}\right\}
$$


When $\sum_{i=1}^{n} \min \left\{\bar{r}_{i}^{\prime}, \bar{s}_{i}^{\prime}\right\} \leq \sigma \leq \min \{|\bar{R}|,|\bar{S}|\}$, there exist $R=\left(r_{1}, \ldots, r_{m}\right), S=\left(s_{1}, \ldots, s_{n}\right)$ satisfying that $|R|=|S|=\sigma$ and for all $1 \leq i \leq n$,

$$
\min \left\{\bar{r}_{i}^{\prime}, \bar{s}_{i}^{\prime}\right\} \leq r_{i}^{\prime} \leq \bar{r}_{i}^{\prime}, \min \left\{\bar{r}_{i}^{\prime}, \bar{s}_{i}^{\prime}\right\} \leq s_{i}^{\prime} \leq \bar{s}_{i}^{\prime} .
$$

In this case there exists a matrix $A \in \mathcal{U}(R, S)$ such that there exists exactly one diagonal of $A$ formed by $\min \left\{\bar{r}_{1}^{\prime}, \bar{s}_{1}^{\prime}\right\}, \ldots, \min \left\{\bar{r}_{n}^{\prime}, \bar{s}_{n}^{\prime}\right\}$. Thus, the equality in (4.10) can be achieved.

When $m=n, \bar{R}=\bar{S}=(1, \ldots, 1) \in \mathbb{R}^{n}$, and $0 \leq \sigma \leq n, \Delta(\bar{R}, \bar{S}, \sigma)$ is the set of all doubly substochastic matrices with total sum equal to $\sigma$. By Lemma 4.2, for all $A \in \Delta(\bar{R}, \bar{S}, \sigma), \operatorname{per}(A) \leq 1$, where $\operatorname{per}(A)=1$ if and only if $\sigma=n$ and $A$ is a permutation matrix ([10,21]).

Lemma 4.3. Let $X=\left(x_{1}, \ldots, x_{n}\right), \bar{X}=\left(\bar{x}_{1}, \ldots, \bar{x}_{n}\right)$ be nonnegative vectors satisfying that $0 \leq x_{i} \leq \bar{x}_{i}$ for all $1 \leq i \leq n, \bar{x}_{1} \leq \ldots \leq \bar{x}_{n}$ and $|X|=x_{1}+\cdots+x_{n}=\sigma$ which is fixed. Let $1 \leq i_{\sigma} \leq n$ satisfying that

$$
\bar{x}_{i_{\sigma}}<\frac{\sigma-\sum_{i=1}^{i_{\sigma}-1} \bar{x}_{i}}{n-i_{\sigma}+1}, \bar{x}_{i_{\sigma}+1} \geq \frac{\sigma-\sum_{i=1}^{i_{\sigma}} \bar{x}_{i}}{n-i_{\sigma}} .
$$

Then

$$
\prod_{i=1}^{n} x_{i} \leq \prod_{i=1}^{i_{\sigma}} \bar{x}_{i}\left(\frac{\sigma-\sum_{i=1}^{i_{\sigma}} \bar{x}_{i}}{n-i_{\sigma}}\right)^{n-i_{\sigma}} .
$$

Proof. If $x_{1}=0$, then $\prod_{i=1}^{n} x_{i}=0$ and (4.11) holds. If $x_{1}=\bar{x}_{1}>0$, then cancel $x_{1}$ from both sides of (4.11) and consider $X^{\prime}=\left(x_{2}, \ldots, x_{n}\right), \bar{X}^{\prime}=\left(\bar{x}_{2}, \ldots, \bar{x}_{n}\right),\left|X^{\prime}\right|=x_{2}+\cdots+x_{n}=\sigma-x_{1}$. Thus without loss of generality, we suppose $0<x_{1}<\bar{x}_{1}$ and first prove the following inequality

$$
\prod_{i=1}^{i_{\sigma}} \bar{x}_{i}\left(\frac{\sigma-\sum_{i=1}^{i_{\sigma}} \bar{x}_{i}}{n-i_{\sigma}}\right)^{n-i_{\sigma}}>x_{1} \prod_{i=2}^{i_{\sigma}} \bar{x}_{i}\left(\frac{\sigma-x_{1}-\sum_{i=2}^{i_{\sigma}} \bar{x}_{i}}{n-i_{\sigma}}\right)^{n-i_{\sigma}},
$$

which is equivalent to

$$
\frac{\bar{x}_{1}}{x_{1}}>\left(\frac{\sigma-x_{1}-\sum_{i=2}^{i_{\sigma}} \bar{x}_{i}}{\sigma-\sum_{i=1}^{i_{\sigma}} \bar{x}_{i}}\right)^{n-i_{\sigma}} .
$$

To show (4.13), we consider the function $f(x)=\ln x+\left(n-i_{\sigma}\right) \ln \left(\sigma-\sum_{i=2}^{i_{\sigma}} \bar{x}_{i}-x\right)$, where $x \in\left(0, \bar{x}_{1}\right]$. Since

$$
f^{\prime}(x)=\frac{1}{x}-\frac{n-i_{\sigma}}{\sigma-\sum_{i=2}^{i_{\sigma}} \bar{x}_{i}-x},
$$

and

$$
f^{\prime \prime}(x)=-\frac{1}{x^{2}}-\frac{n-i_{\sigma}}{\left(\sigma-\sum_{i=2}^{i_{\sigma}} \bar{x}_{i}-x\right)^{2}}<0,
$$

$f^{\prime}(x)$ is decreasing on $\left(0, \bar{x}_{1}\right]$. Thus for $x \in\left(0, \bar{x}_{1}\right)$,

$$
\begin{aligned}
f^{\prime}(x) & >f^{\prime}\left(\bar{x}_{1}\right)=\frac{1}{\bar{x}_{1}}-\frac{n-i_{\sigma}}{\sigma-\sum_{i=1}^{i_{\sigma}} \bar{x}_{i}} \\
& =\frac{\sigma-\sum_{i=1}^{i_{\sigma}} \bar{x}_{i}-\left(n-i_{\sigma}\right) \bar{x}_{1}}{\bar{x}_{1}\left(\sigma-\sum_{i=1}^{i_{\sigma}} \bar{x}_{i}\right)} \geq \frac{\left(n-i_{\sigma}\right)\left(\bar{x}_{i_{\sigma}}-\bar{x}_{1}\right)}{\bar{x}_{1}\left(\sigma-\sum_{i=1}^{i_{\sigma}} \bar{x}_{i}\right)} \geq 0 .
\end{aligned}
$$


Electronic Journal of Linear Algebra, ISSN 1081-3810

A publication of the International Linear Algebra Society

Volume 37, pp. 256-271, March 2021.

Therefore, $f(x)$ is increasing on $\left(0, \bar{x}_{1}\right]$, which implies that $f\left(x_{1}\right)<f\left(\bar{x}_{1}\right)$ if $x_{1}<\bar{x}_{1}$, i.e.

$$
\ln \bar{x}_{1}+\left(n-i_{\sigma}\right) \ln \left(\sigma-\sum_{i=2}^{i_{\sigma}} \bar{x}_{i}-\bar{x}_{1}\right) \geq \ln x_{1}+\left(n-i_{\sigma}\right) \ln \left(\sigma-\sum_{i=2}^{i_{\sigma}} \bar{x}_{i}-x_{1}\right) .
$$

From the above inequality, we can easily obtain (4.13). Thus (4.12) holds and we have

$$
\begin{aligned}
\prod_{i=1}^{i_{\sigma}} \bar{x}_{i}\left(\frac{\sigma-\sum_{i=1}^{i_{\sigma}} \bar{x}_{i}}{n-i_{\sigma}}\right)^{n-i_{\sigma}} & >x_{1} \prod_{i=2}^{i_{\sigma}} \bar{x}_{i}\left(\frac{\sigma-x_{1}-\sum_{i=2}^{i_{\sigma}} \bar{x}_{i}}{n-i_{\sigma}}\right)^{n-i_{\sigma}} \\
& >x_{1} x_{2} \prod_{i=3}^{i_{\sigma}} \bar{x}_{i}\left(\frac{\sigma-x_{1}-x_{2}-\sum_{i=3}^{i_{\sigma}} \bar{x}_{i}}{n-i_{\sigma}}\right)^{n-i_{\sigma}} \\
& >\cdots>\prod_{i=1}^{i_{\sigma}} x_{i}\left(\frac{\sigma-\sum_{i=1}^{i_{\sigma}} x_{i}}{n-i_{\sigma}}\right)^{n-i_{\sigma}}
\end{aligned}
$$

By the arithmetic-geometric inequality,

$$
\left(\frac{\sigma-\sum_{i=1}^{i_{\sigma}} x_{i}}{n-i_{\sigma}}\right)^{n-i_{\sigma}} \geq x_{i_{\sigma}+1} \cdots x_{n}
$$

Thus, the lemma holds.

For any $A \in \Delta(\bar{R}, \bar{S}, \sigma)$, by Lemma 4.2

$$
\operatorname{per}(A) \leq \max \left\{\prod_{i=1}^{n} \min \left\{r_{i}^{\prime}, s_{i}^{\prime}\right\}:|R|=|S|=\sigma, R \triangleleft \bar{R}, S \triangleleft \bar{S}\right\} .
$$

For all $1 \leq i \leq n, \min \left\{r_{i}^{\prime}, s_{i}^{\prime}\right\} \leq \bar{t}_{i}$ where $\bar{t}_{i}=\min \left\{\bar{r}_{i}^{\prime}, \bar{s}_{i}^{\prime}\right\}$. Note that $\sum_{i=1}^{n} \min \left\{r_{i}^{\prime}, s_{i}^{\prime}\right\} \leq \sigma$. Lemma 4.2 and Lemma 4.3 imply the following theorem.

THEOREM 4.4. Given nonnegative vectors $\bar{R}=\left(\bar{r}_{1}, \ldots, \bar{r}_{n}\right), \bar{S}=\left(\bar{s}_{1}, \ldots, \bar{s}_{n}\right), 0 \leq \sigma \leq \min \{|\bar{R}|,|\bar{S}|\}$, let $\bar{t}_{i}=\min \left\{\bar{r}_{i}^{\prime}, \bar{s}_{i}^{\prime}\right\}$ and $A$ be a matrix in $\Delta(\bar{R}, \bar{S}, \sigma)$.

1. If $0 \leq \sigma \leq \sum_{i=1}^{n} \bar{t}_{i}, 0 \leq i_{\sigma} \leq n$ such that for $i_{\sigma} \neq 0$,

$$
\bar{t}_{i_{\sigma}}<\frac{\sigma-\sum_{i=1}^{i_{\sigma}-1} \bar{t}_{i}}{n-i_{\sigma}+1}, \bar{t}_{i_{\sigma}+1} \geq \frac{\sigma-\sum_{i=1}^{i_{\sigma}} \bar{t}_{i}}{n-i_{\sigma}}
$$

and set $i_{\sigma}=0$ when $\bar{t}_{1} \geq \frac{\sigma}{n}$, then

$$
\operatorname{per}(A) \leq \begin{cases}\prod_{i=1}^{i_{\sigma}} \bar{t}_{i}\left(\frac{\sigma-\sum_{i=1}^{i_{\sigma}} \bar{t}_{i}}{n-i_{\sigma}}\right)^{n-i_{\sigma}} & \text { if } i_{\sigma} \neq 0 \\ \left(\frac{\sigma}{n}\right)^{n} & \text { if } i_{\sigma}=0\end{cases}
$$

The equality holds when $A=\left[a_{i, j}\right]$ is a diagonal matrix such that if $i_{\sigma} \neq 0$, then

$$
a_{i, i}= \begin{cases}\bar{t}_{i} & \text { if } 1 \leq i \leq i_{\sigma} \\ \frac{\sigma-\sum_{i=1}^{i_{\sigma}} \bar{t}_{i}}{n-i_{\sigma}} & \text { if } i_{\sigma}+1 \leq i \leq n\end{cases}
$$


and if $i_{\sigma}=0$, then

$$
a_{i, i}=\frac{\sigma}{n} \text { for } 1 \leq i \leq n .
$$

2. If $\sum_{i=1}^{n} \bar{t}_{i} \leq \sigma \leq \min \{|\bar{R}|,|\bar{S}|\}$, then

$$
\operatorname{per}(A) \leq \prod_{i=1}^{n} \bar{t}_{i}
$$

The equality holds when $A$ is in $\mathfrak{E}(R, S)$ where $R \triangleleft \bar{R}, S \triangleleft \bar{S},|R|=|S|=\sigma$, and there exist two rearrangements $i_{1}, \ldots, i_{n} ; j_{1}, \ldots, j_{n}$ of $1, \ldots, n$ such that

$$
a_{i_{k}, j_{k}}=\min \left\{\bar{r}_{i}^{\prime}, \bar{s}_{i}^{\prime}\right\}=\bar{t}_{i},(k, i=1, \ldots, n) .
$$

Corollary 4.5. For $0 \leq s \leq n$ and $A \in \omega_{n}^{s}$,

$$
\operatorname{per}(A) \leq\left(\frac{s}{n}\right)^{n}
$$

Acknowledgment. We thank the anonymous referees very much for their valuable suggestions and comments, which greatly help us to improve the results of this paper.

\section{REFERENCES}

[1] G. Birkhoff. Tres observaciones sobre el algebra lineal. Univ. Nac. Tucumán, Revista, Ser. A, 5:147-151, 1946.

[2] E.D. Bolker. Transportation polytopes. J. Combin. Theory Ser. B, 13(3):251-262, 1972.

[3] R.A. Brualdi. Convex sets of non-negative matrices. Canad. J. Math., 20(1):144-157, 1968.

[4] R.A. Brualdi. Combinatorial properties of symmetric non-negative matrices. Colloquio Internazionale sulle Theorie Combinatorie, Roma, 1973, Acad. Naz. Lincei, Rome, Tomo II(17):99-120, 1976.

[5] R.A. Brualdi. Combinatorial Matrix Classes. Encyclopedia of Mathematics and its Applications, 108, Cambridge University Press, 2006.

[6] R.A. Brualdi and L. Cao. Symmetric, Hankel-symmetric, and Centrosymmetric doubly stochastic matrices. Acta Math. Vietnam, 43(4):675-700, 2018.

[7] A.B. Cruse. Some combinatorial properties of centrosymmetric matrices. Linear Algebra Appl., 16:65-77, 1977.

[8] L. Cao, S. Koyuncu, and T. Parmer. A mininal completion of doubly substochastic matrix. Linear Multilinear Algebra, 64(11):2313-2334, 2016.

[9] L. Cao and Z. Chen. Partitions of the polytope of doubly substochastic matrices. Linear Algebra Appl., 563:98-122, 2019.

[10] L. Cao, Z. Chen, S. Koyuncu, and H. Li. Permanents of doubly substochastic matrices. Linear Multilinear Algebra, 68(3):594-605, 2020.

[11] Z. Chen, L. Cao, and Q.-W. Wang. The extreme points of certain polytopes of doubly substochastic matrices. Linear Multilinear Algebra, 68(10):1956-1971, 2020.

[12] S.-J. Cho and Y.-S. Nam. Convex polytopes of generalized doubly stochastic matrices. Commun. Korean Math. Soc., 4(16):679-690, 2001.

[13] G.B. Dantzig. Linear Programming and Extensions. Princeton University Press, Princeton, N.J., 1962.

[14] A.L. Dulmage and N.S. Mendelsohn. The term and stochastic ranks of a matrix. Canad. J. Math., 11(11):269-279, 1959.

[15] D.R. Fulkerson. Hitchcock transportation problem. Rand. Corp. Report, P-890, 1956.

[16] D.A. Gregory, S.J. Kirkland, and N.J. Pullman. Row-stochastic matrices with a common left fixed vector. Linear Algebra Appl., 169:131-149, 1992.

[17] W.B. Jurkat and H.J. Ryser. Term ranks and permanents of nonnegative matrices. J. Algebra, 5(3):342-357, 1967.

[18] M. Katz. On the extreme points of a certain convex polytope. J. Combinatorial Theory, 8(4):417-423, 1970.

[19] M. Katz. On the extreme points of the set of substochastic and symmetric matrices. J. Math. Anal. Appl., 37:576-579, 1972 . 
[20] V. Klee and C. Witzgall. Facets and vertices of transportation polytope. Mathematics of the Decision Sciences, Part I (Seminar, Stanford, Calif., 1967), American Mathematical Society, Providence, 257-282, 1968.

[21] M. Marcus and M. Newman. On the minimun of the permanent of a doubly stochastics matrix. Duke Math. J., 26(1):61-72, 1959.

[22] L. Mirsky. Proofs of two theorems on doubly stochastics matrices. Proc. Amer. Math. Soc, 9(3):371-374, 1958.

[23] L. Mirsky. On a convex set of matrices. Arch. Math., 10(1):88-92, 1959. 